\section{Imaging of salvaged myocardium after acute myocardial infarction}

A multinational study has found that cardiovascular magnetic resonance imaging (CMR) can differentiate between reversible and irreversible cardiac damage after acute myocardial infarction, thereby permitting quantification of the salvaged area after reperfusion.

Friedrich et al. enrolled 92 patients with acute myocardial infarction and 18 healthy controls. The median time from symptom onset to reperfusion was $6 \mathrm{~h}$, and CMR was carried out a median of 3 days after the occurrence of infarction. T2-weighted CMR was used to visualize myocardial edema, and contrastenhanced ('late-enhancement') CMR was used to measure the area of tissue necrosis, with gadolinium DTPA as the contrast medium.

High T2 signal abnormality matched the location of late enhancement for all 92 patients; mean size of the high-T2 area-which represents salvageable myocardium-was, however, significantly larger than the necrotic late-enhancement area. The size difference between the high-T2 and late-enhancement areas correlated inversely with time between symptom onset and reperfusion. Although visual examination showed a localized high T2 signal in 2 of the 18 healthy volunteers, quantitative analysis was negative for any abnormality.

This study suggests that CMR can identify injured but viable myocardium in the area at risk after acute myocardial infarction. This information could be valuable in the management of patients with acute myocardial infarction and might provide a novel end point for clinical trials.

Original article Friedrich MG et al. (2008) The salvaged area at risk in reperfused acute myocardial infarction as visualized by cardiovascular magnetic resonance. J Am Coll Cardiol 51: 1581-1587

\section{Long-term outcomes of patients with peripheral arterial disease after vascular surgery}

Aggressive treatment of atherosclerotic risk factors is recommended for patients with peripheral arterial disease (PAD) who require vascular surgery, yet the survival and treatment of these patients after surgery is unknown. Welten et al. conducted an observational study to determine the long-term outcomes of patients with PAD who undergo open vascular surgery.

This study involved 2,730 patients with PAD who underwent major vascular surgery at a medical center in Rotterdam, The Netherlands, during the period January 1993 to June 2006. These patients were propensity matched to patients with coronary artery disease (CAD) who underwent coronary angioplasty at the same center.

Patients with PAD had a significantly higher risk of long-term all-cause and cardiac mortality than those with CAD (unadjusted hazard ratio $2.4,95 \% \mathrm{Cl} 2.18-2.65$; mean follow up $6.37 \pm 4.08$ years and $9.17 \pm 4.14$ years for patients with PAD and CAD, respectively). For patients with PAD, the leading cause of death was cerebrocardiovascular events, in both the short term (i.e. 30 days after surgery; $76 \%$ ) and long term (46\%), yet patients with PAD received significantly fewer cardiac medications-such as statins, $\beta$-blockers, angiotensin-converting-enzyme inhibitors, aspirin and nitrates - than did patients with CAD ( $P<0.001$ for all cardiac medications).

The authors conclude that outcome after surgery is worse in patients with PAD than in those with CAD, and they attribute the poor outcome in patients with PAD to the undertreatment of these individuals.

Original article Welten GMJM et al. (2008) Long-term prognosis of patients with peripheral arterial disease: a comparison in patients with coronary artery disease. J Am Coll Cardiol 51: 1588-1596 